\title{
Statement on Minimum Standards for the Care of Older People in Emergency Departments by the Geriatric Emergency Medicine Special Interest Group of the International Federation for Emergency Medicine
}

\author{
Brittany Ellis, MD, MSc ${ }^{*}$; Christopher R. Carpenter, MD, MSc ${ }^{\ddagger}$; Judy A. Lowthian, PhD, MPH, BAppSc \\ $(\text { SpPath) })^{\S \uparrow}$; Simon P. Mooijaart, MD, PhD"; Christian H. Nickel, MD**; Don Melady, MD, MSc(Ed)*
}

Keywords: geriatric emergency medicine, international, standards, older people

SCOPE

In 2015, the International Federation for Emergency Medicine (IFEM) established a geriatric emergency medicine special interest group, recognizing that older people are the fastest growing demographic population in the majority of countries, both developing and developed. The group was tasked with developing and promoting geriatric emergency medicine, including the establishment of a brief statement outlining minimum standards of care for older people in emergency departments (EDs) around the world. These minimum standards represent a first step on which future activities can be built.

IFEM recognizes that, although these standards are global, the local solutions will be unique because they are dependent upon local, national, and regional factors. This statement applies to older people, a group who cannot be defined by age alone, but instead by the complex interactions of increased age and biopsychosocial vulnerability. Through this Statement of Minimum Standards, it is the goal of IFEM to catalyse change and improve care across emergency medicine for all older patients.

Demographic changes, not limited to the developed world, have created an increase in older adults across all countries. This demographic shift is accompanied by an increased demand on health and social care resources, including EDs. For the well-being of patients and families, as well as national economies, continued improvements in the care of older people in EDs must be a priority for both clinical care and health policy.

For many older people in higher- and lower-resource settings, the ED is an important, if not the only, access point to the healthcare system. EDs function as an intersection point in the continuum of care. They represent a unique opportunity to influence the health experience and outcomes for older people, who place unique demands on healthcare systems. Older people often have complex care needs. They are more likely to be frail and are at increased risk of adverse events and health outcomes. For all older patients, acute illness or injury is a critical moment with an even greater risk of adverse outcomes. Integrated evaluation and management in the ED can lead to improved outcomes for older people not only through optimal immediate care, but also by preventing further morbidity and by providing more efficient and effective services across healthcare systems.

To ensure that the needs of older people are met in every ED, IFEM mandates the following eight minimum standards. These are centred on the recognition that older people are a core population of emergency health service users whose care needs are often different from those of children and younger adults. These standards are

From the *Schwartz-Reisman Emergency Medicine Institute, Mount Sinai Hospital, Toronto, ON; †Department of Emergency Medicine, University of Ottawa, Ottawa, ON; ¥Division of Emergency Medicine and Emergency Care Research Core, Washington University in St. Louis, St. Louis, MO; $\S$ School of Public Health \& Preventative Medicine, Monash University, Melbourne, Australia; đBolton Clarke Research Institute, Bolton Clarke, St. Kilda, Australia; IDepartment of Gerontology and Geriatrics, Leiden University Medical Centre, Leiden, Netherlands; and the **Department of Emergency Medicine, University Hospital Basel, Basel, Switzerland.

Correspondence to: Dr. Brittany Ellis, Mount Sinai Hospital, 600 University Avenue, Room 206, Toronto, ONT M5G 1X5, Canada; Email: brittanyjellis@gmail.com

A previous error in this article has been corrected, see 10.1017/cem.2018.39. 
the product of interdisciplinary and international collaboration, using the IFEM Framework for Quality and Safety in the Emergency Department. ${ }^{1}$ The standards can be used to guide the care of older people in EDs and national health systems across the globe.

1) The right approach: EDs should recognize that older people are a core population of service users, not defined by chronological age but by the complex interactions of physical, psychological and social frailty, disease, and access to care.

2) The right personnel: EDs should identify that adequate care of older people often involves an interdisciplinary and multi-specialty approach to their interconnected medical, social, cognitive, and functional issues, and should have appropriately trained interdisciplinary staff.

3) The right environment: EDs should address safety and accessibility issues for older people with the design of the physical environment and available equipment.

4) The right decision making: EDs should ensure that the needs and goals of older people are considered at all levels of decision making, recognizing the importance of shared decision making in this age group.
5) The right processes: EDs should establish policies and procedures that guide holistic person-centred management of problems common to older people, and should promote quality improvement focusing on older people.

6) The right support: EDs should facilitate education about the physical, social, and functional issues common to older people, using evidence-based principles in staff training and development.

7) The right results: EDs should actively support patient-centred care that leads to optimal outcomes for older people.

8) The right system: EDs should include strategies for safe and effective transitions of care of older people with support from community- and hospital-based services.

Competing interests: None declared.

\section{REFERENCE}

1. International Federation for Emergency Medicine. Framework for quality and safety in the emergency department; 2012. Available at: http://www.ifem.cc/Resources/Policies andGuidelines.aspx. 